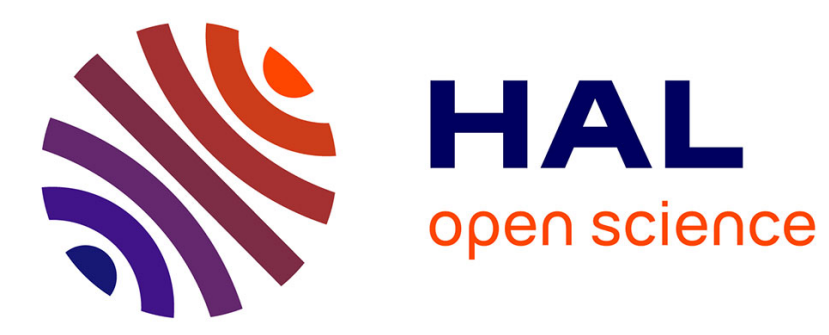

\title{
Application of scanning electron diffraction in the transmission electron microscope for the characterisation of dislocations in minerals
}

Billy Clitton Nzogang, Alexandre Mussi, Patrick Cordier

\section{- To cite this version:}

Billy Clitton Nzogang, Alexandre Mussi, Patrick Cordier. Application of scanning electron diffraction in the transmission electron microscope for the characterisation of dislocations in minerals. Mineralogical Magazine, 2019, Mineralogical Magazine, 83 (1), pp.71-79. 10.1180/mgm.2018.144 . hal02094470

\section{HAL Id: hal-02094470 \\ https://hal.univ-lille.fr/hal-02094470}

Submitted on 6 Jun 2019

HAL is a multi-disciplinary open access archive for the deposit and dissemination of scientific research documents, whether they are published or not. The documents may come from teaching and research institutions in France or abroad, or from public or private research centers.
L'archive ouverte pluridisciplinaire HAL, est destinée au dépôt et à la diffusion de documents scientifiques de niveau recherche, publiés ou non, émanant des établissements d'enseignement et de recherche français ou étrangers, des laboratoires publics ou privés. 


\title{
Application of Scanning Electron Diffraction in the Transmission Electron Microscope for the characterization of dislocations in minerals
}

\author{
Billy C. Nzogang, Alexandre Mussi and Patrick Cordier*
}

Univ. Lille, CNRS, INRA, ENSCL, UMR 8207 - UMET - Unité Matériaux Et Transformations, F-59000 Lille, France.

\section{Cite as:}

B.C. Nzogang, A. Mussi \& P. Cordier (2019) Application of Scanning Electron Diffraction in the Transmission Electron Microscope for the characterization of dislocations in minerals. Mineralogical Magazine, 83(1), 71-79, https://doi.org/10.1180/mgm.2018.144

\begin{abstract}
We present an application of scanning electron diffraction for the characterization of crystal defects in olivine, quartz and phase $A$. In this mode, which takes advantage of the ASTAR ${ }^{T M}$ module from NanoMEGAS, a slightly convergent probe is scanned over the sample with a short acquisition time (a few tens of ms) and the spot patterns are acquired and stored for further post-processing. Originally, orientation maps were constructed from automatic indexing at each probe location. Here we present another application where images are reconstructed from the intensity of diffraction spots, producing either so-called "virtual" bright- or dark field images. We show that these images present all the characteristics of contrast (perfect crystal or defects) of conventional TEM images. Data being acquired with a very short time per probe location (a few tens of milliseconds), this technique appears very attractive for the characterization of beam-sensitive materials. However, since the acquisition is done at a given orientation, fine tuning of the diffraction conditions at a given location for each reflexion is not possible. This might be a difficulty for some precise, quantitative contrast analysis.
\end{abstract}

Keywords: Transmission electron microscopy, scanning electron diffraction; dislocations.

\footnotetext{
* Corresponding author: Patrick Cordier (patrick.cordier@univ-lille1.fr, ORCID: 0000-0002-1883-2994)
} 


\section{Introduction}

The concept of crystal dislocation as a topological defect able to account for plastic deformation of solids has been proposed independently by Egon Orowan, Michael Polanyi and Geoffrey Ingram Taylor in 1934. The first direct observation of those defects in a transmission electron microscope (TEM) by Hirsch et al. in 1956 had a great influence in the development of the subject with applications to mechanical properties and other fields like crystal growth phenomena. In 1965, McLaren and Phakey published a series of papers showing the first TEM characterizations of defects (including dislocations) in an important rock-forming mineral: quartz. From the beginning, the development of the diffraction contrast technique was central, and it is still more widely used than, for instance, the high-resolution imaging of the dislocation cores. The so-called conventional TEM, which is based on diffraction contrast, has the key advantage to provide information at the mesoscale of dislocation arrangements and patterning, which plays a key role in plasticity. The diffraction pattern, which forms in the back focal plane of a transmission electron microscope, is used to guide specimen tilting so as to orientate a chosen diffracting plane in Bragg position (Fig. 1a). Due to the elastic distortions, these diffraction conditions are locally altered close to the dislocation lines giving rise to a strong contrast in bright- or dark-field images (Hirsch et al., 1956). Cockayne et al. (1965) have shown that tilting slightly away from exact Bragg conditions (so as for instance to excite higher order reflexions) dramatically increases the resolution of the image of the dislocation lines, this is the so-called weak-beam dark-field (WBDF) mode. A major further development was to show that these strict diffracting conditions could be preserved along a wide range of tilt angles to allow electron tomography of dislocations to be performed (Barnard et al., 2006a,b, 2010; Mussi et al., 2014) to obtain a true three-dimensional (3D) reconstruction of a dislocation network. In all these techniques, image formations, as well as most operations, are performed at the microscope, and final images are captured by a detector conjugate to the image plate. In case of beam-sensitive materials, the time necessary for adjustments and image optimization might be detrimental and lead to damage the sample before the characterizations are fulfilled. Quartz, as originally pointed out by McLaren and Phakey (1965b), is the archetypal case of a beam-sensitive mineral, but this behaviour is exhibited by several other important rock-forming minerals (e.g. topaz, albite, nepheline, phyllosilicates) and is now met for the study of most high-pressure phases which are metastable under ambient conditions.

Here, following the proposition of Rauch and Véron (2014a) and Kiss et al. (2016) we present an alternative technique for dislocation imaging and characterization in the TEM based on scanning electron diffraction (SED), which consists in recording a data cube of diffraction patterns from which imaging is extracted off-line by post-treatment. An application on two important rock-forming minerals, olivine and quartz, and on a high-pressure hydrated phase (phase A) are presented. 


\section{Experimental}

Three samples have been used in this study. One is a natural olivine $\left((\mathrm{Mg}, \mathrm{Fe})_{2} \mathrm{SiO}_{4}\right)$ deformed experimentally by M. Thieme and S. Demouchy (Geosciences Montpellier, France) at $1030^{\circ} \mathrm{C}(\mathrm{CMT} 16-8)$ in a gas-medium apparatus (Thieme et al., 2018). The second is a natural quartz from a granulite (Bohemian massif). The third is a synthetic sample of a dense hydrous magnesium silicate (DHMS), phase A. For all samples, thin sections have been cut and mechanically polished down to a thickness of about $30 \mu \mathrm{m}$. In order to reach electron transparency, the sample foils were Ar-ion sputtered with a Gatan ${ }^{\circledR}$ DuoMill ${ }^{\mathrm{TM}}$ model 600 . Carbon films were deposited on the thin foils to ensure electron conduction.
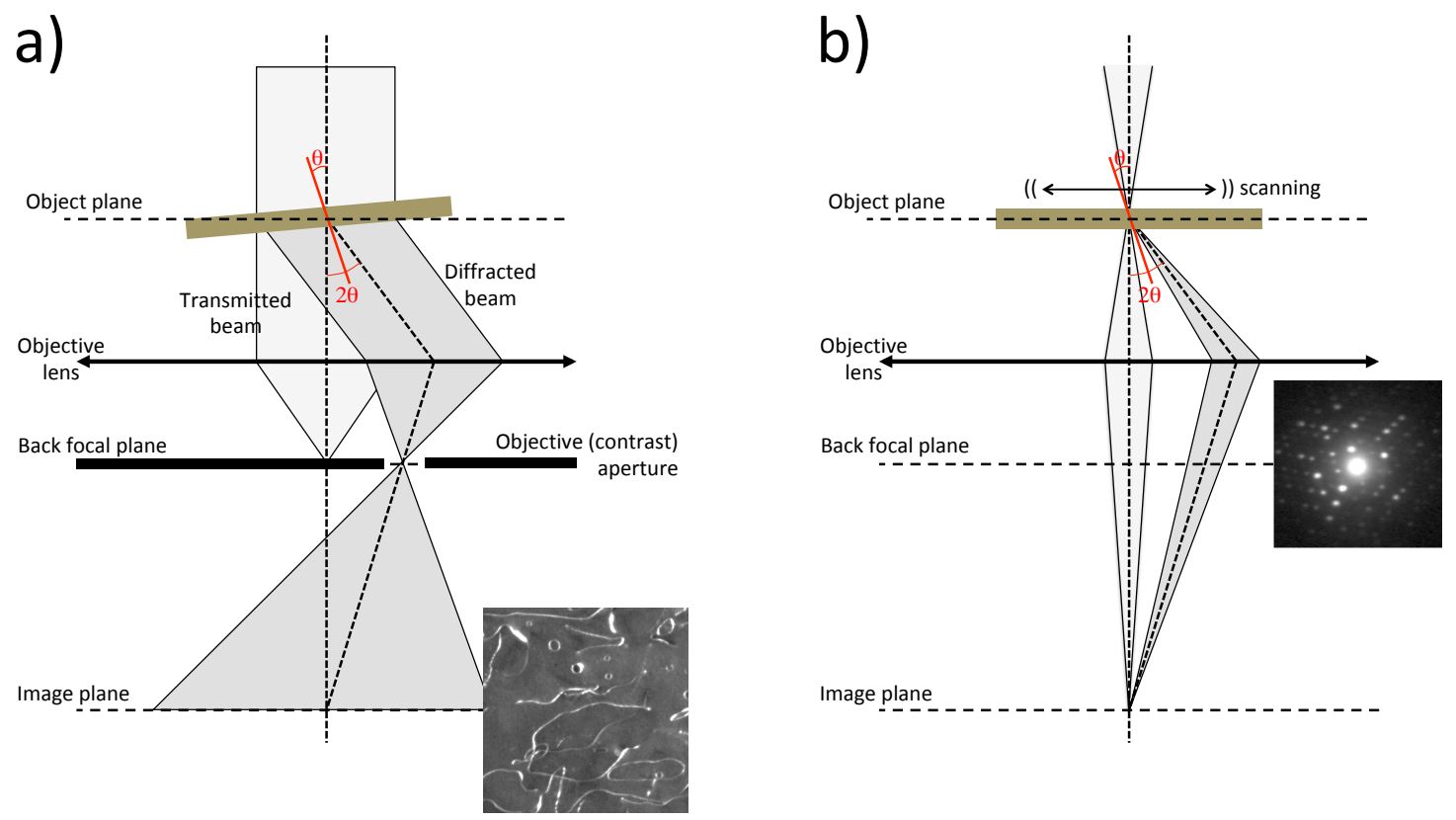

Figure 1: Microscope settings

a) Conventional TEM dark-field. The electron beam is parallel. The sample is tilted to orientate a given diffraction plane close to Bragg conditions. A contrast aperture is used in the back focal plane so as to select the diffracted beam to form the image that is recorded. To perform a weak-beam dark-field the specimen is slightly tilted away from the exact Bragg conditions.

b) Scanning electron diffraction. The microscope is set in microprobe mode (the electron beam has a small convergence) and the spot is scanned over the sample. Only the diffraction patterns are recorded and stored.

TEM investigations were performed with a $\mathrm{FEI}^{\circledR}$ Tecnaï $\mathrm{G}^{2} 20$ Twin microscope, operating at $200 \mathrm{kV}$ and with a Philips CM30 microscope operating at $300 \mathrm{kV}$, both equipped with a $\mathrm{LaB}_{6}$ filament and using a double tilt sample-holder.

Orientation maps were acquired with a step size of $2 \mathrm{~nm}$ using SED in the TEM with the ASTAR ${ }^{\mathrm{TM}}$ tool from NanoMEGAS (Rauch and Véron, 2014b). The TEM is set in micro-beam mode (spot size $1 \mathrm{~nm}$, fluence rate $3.5 \times 10^{5}$ electron. $\mathrm{nm}^{-2} \mathrm{~s}^{-1}$ ) and the incident electron beam, which is focused on the sample, is scanned over 
the area thanks to a dedicated hardware control system of the TEM deflecting coils (Fig. 1b). In this mode where the sample is illuminated by a convergent beam, the diffraction pattern consists in discs. Using the smallest condenser aperture $(50 \mu \mathrm{m})$ the diffraction patterns are made of very small spot-like discs (Fig. 1b) that can be indexed as spot patterns. The individual exposure time at each location is a few tens of milliseconds. Diffraction patterns are collected with an external Stingray CCD camera that points on the TEM phosphorous screen and stored in the computer memory for further indexation and post processing. The diffraction patterns are collected as $144 \times 144$ pixels at a camera length of 105,135 or $175 \mathrm{~mm}$. In the ASTAR system, electron diffraction spot patterns are indexed (providing the local crystal orientations) by comparing individually obtained patterns via cross-correlation matching techniques with pre-calculated electron diffraction templates generated every $1^{\circ}$ (orientation resolution). From this indexation, one usually obtains an orientation map which displays, with a colour coding, the most probable orientation at each scanned beam position. Here we present another use of diffraction data cubes. From experimental spot patterns, virtual bright-field (VBF) maps can be generated, which represents the fluctuation of the central beam intensity of diffraction patterns. This builds up a STEM-like brightfield contrast directly related to the orientation maps. Virtual dark-field (VDF) images can also be generated by reproducing the same operation with a selected diffracting spot.

\section{Observations and discussion}

\section{Olivine}

Olivine is the major component of the Earth's mantle down to $410 \mathrm{~km}$ depth. This phase is stable at ambient pressure and also quite stable under the electron beam. In Fig. 2, we present ten VDF images reconstructed from the $002,00 \overline{2}, 004,00 \overline{4}, 2 \overline{4} 0, \overline{2} 40,1 \overline{2} \overline{2}, \overline{1} 22,1 \overline{2} 2$, and $\overline{1} 2 \overline{2}$ diffraction spots present in the $1,440,000$ diffraction patterns of the data cube, which are close to the [210] zone axis (in the Pbnm space group). All images are extracted from the same set of diffraction patterns recorded under steady conditions, in particular at a constant orientation of the specimen in the microscope. One can see however that local diffraction conditions are different for each VDF image. For instance, with 002, exact Bragg conditions $(s=0)$ are fulfilled within a wide band toward the top of the image which corresponds to larger Bragg deviation (hence to higher resolution WBDF image) with $00 \overline{2}$ (Fig. 2d). In the latter image, the best conditions for observation are found in the right and upper parts. Figs $2 \mathrm{e}$ and $2 \mathrm{f}$ present VDF reconstructed with 004 and $00 \overline{4}$. These reflexions have a larger structure factor than 002 (1384 versus 373). The information gained are in principle the same but the quality of the images is higher. The location of exact Bragg position $s=0$ is also slightly different on each image. The following images are reconstructed with 240 and 122 reflexions. Their structure factors are comparable in olivine (328 and 312 respectively). These images allow identifying the dislocations present. Indeed, in orthorhombic olivine, only two different dislocation types exist with [100] and [001] Burgers vectors (in the Pbnm space group). 


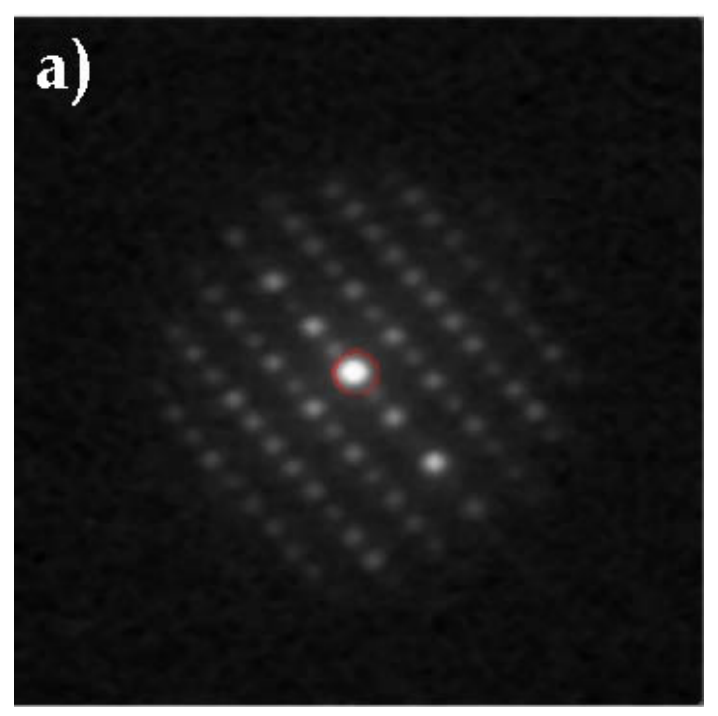

b)
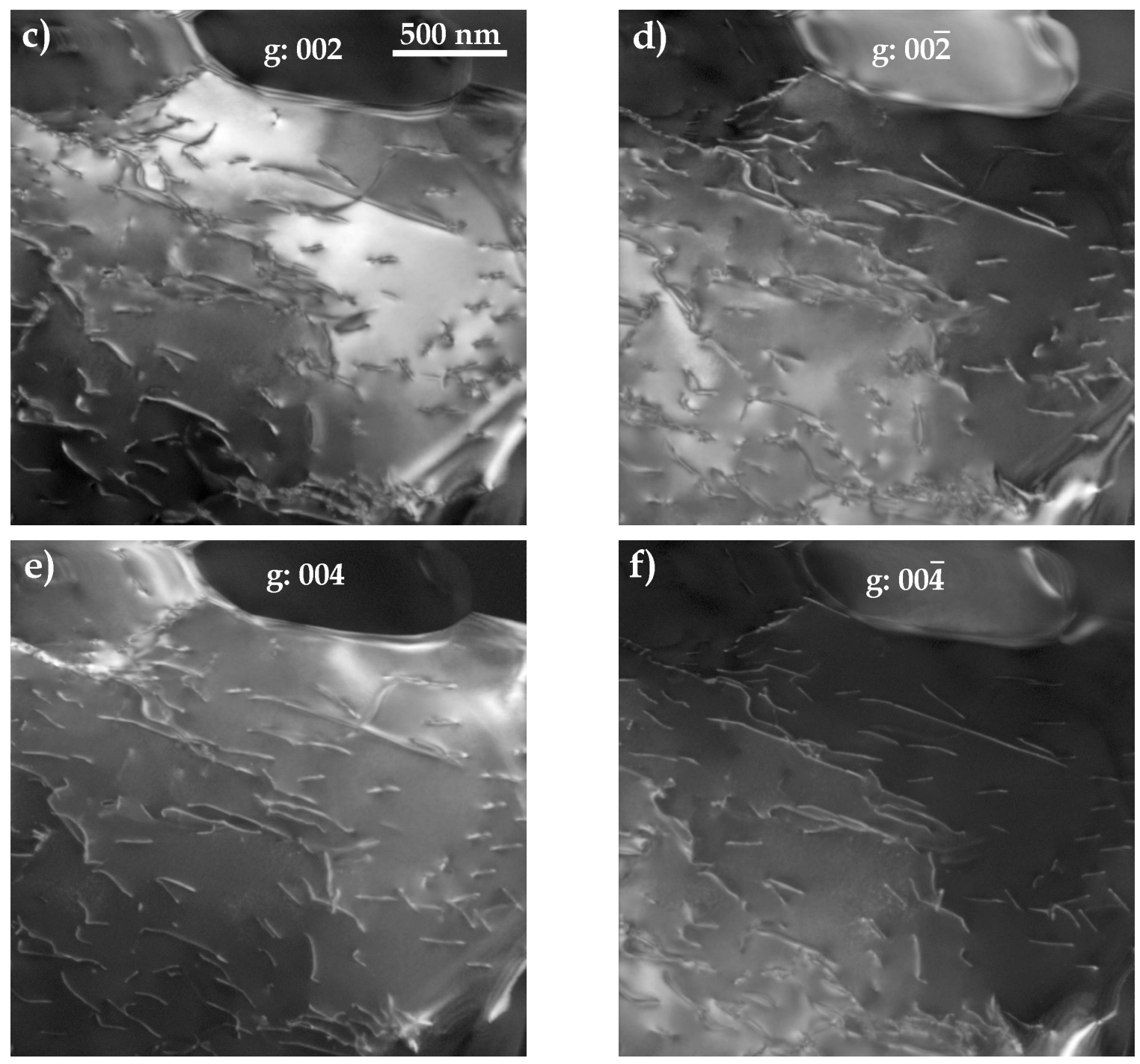

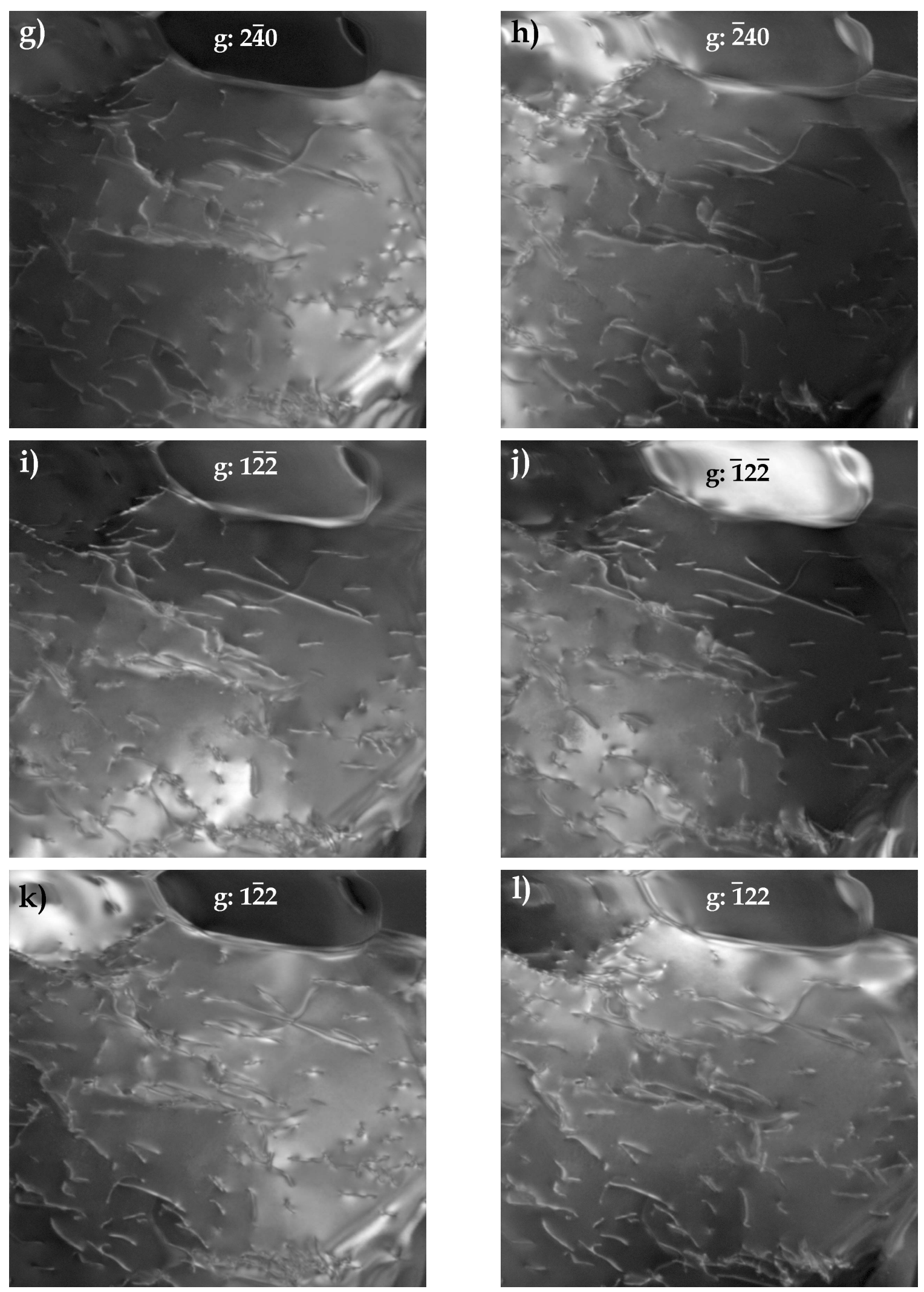
Figure 2 (above): Olivine - Acquisition parameters: spot size $1 \mathrm{~nm}$; step size $2 \mathrm{~nm}$; acquisition time $10 \mathrm{~ms} ; 1200 \times 1200$ points.

a) Diffraction pattern. The sample is oriented close to the [210] zone axis.

b) Simulation of the diffraction pattern with Electron Diffraction. The spot diameter indicates the intensity.

c-l) Virtual dark-fields: the diffraction vector is indicated on the pictures.

Dislocation identification could be performed by comparing images reconstructed with 004 (which images only [001] dislocations) and with 240 (which images only [100] dislocations). However, on VDF reconstructed with $2 \overline{4} 0$ and $\overline{2} 40$, deviation from exact Bragg position is not sufficient to reach weak-beam conditions, and residual contrasts on [001] dislocations give ambiguous information. Identification can be performed however by comparing images reconstructed with $00 \overline{4}$ and $\overline{1} 2 \overline{2}$ reflexions. Figure 3 displays enlargements of Fig. 2 which allow to clearly identify [001] dislocations (seen on Fig. 3a and 3b) from a [100] dislocations (visible on Fig. 3b only). Fig. 3c shows the intensity profile of a [001] dislocation in Fig. 3a. The full width at half maximum is about $12 \mathrm{~nm}$ demonstrating that resolution comparable to WBDF can be attained by SED. With $\overline{1} 2 \overline{2}$, images of [100] and [001] dislocations correspond to $\boldsymbol{g} . \boldsymbol{b}$ products of 1 and 2 respectively. The intensity profile of Fig. 3d across two dislocations shows that indeed, the intensity of the [001] dislocation is larger, but only by a factor of 1.63 which is lower than the 2:1 ratio expected. The SED is thus able to lead to dislocation Burgers vectors characterizations through a single data acquisition only.
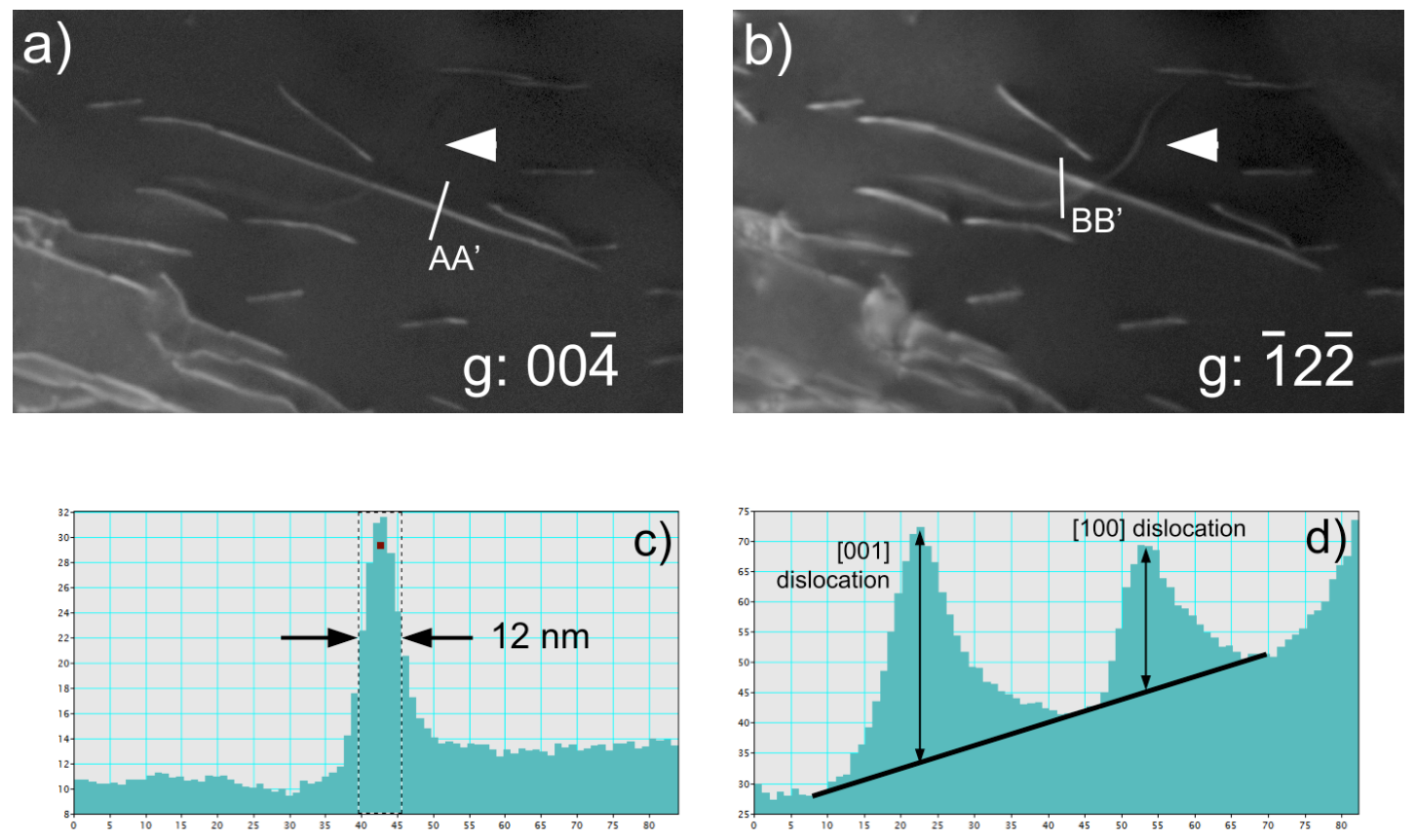

Figure 3: Olivine - Burgers vectors determinations on enlargements of Figure 2.

a) With $00 \overline{4}$, only [001] dislocations are in contrast

b) With $\overline{1} 2 \overline{2}$, all dislocations are visible

c) Intensity profile along the AA' line from Fig $3 a$. The width of the dislocation image is about $12 \mathrm{~nm}$.

d) Intensity profile along the BB' line from Fig $3 b$ showing the respective intensity of [100] and [001] dislocations. 


\section{Quartz}

In its low-temperature form ( $\alpha$-quartz) quartz is the most common silica phase. It is ubiquitous in the Earth's crust where it is, after feldspars, the most abundant phase. From the sixties, numerous studies have addressed the role of defects in the mechanical properties of quartz, however, the detailed characterization of crystal defects were always limited by the rapid amorphisation of the samples under the electron beam (McLaren and Phakey, 1965b). Some alternative TEM techniques like large-angle convergent-beam electron diffraction (Cordier et al., 1995) offer now the possibility to carry out detailed characterizations, but the operating conditions are detrimental to observation in the real space.
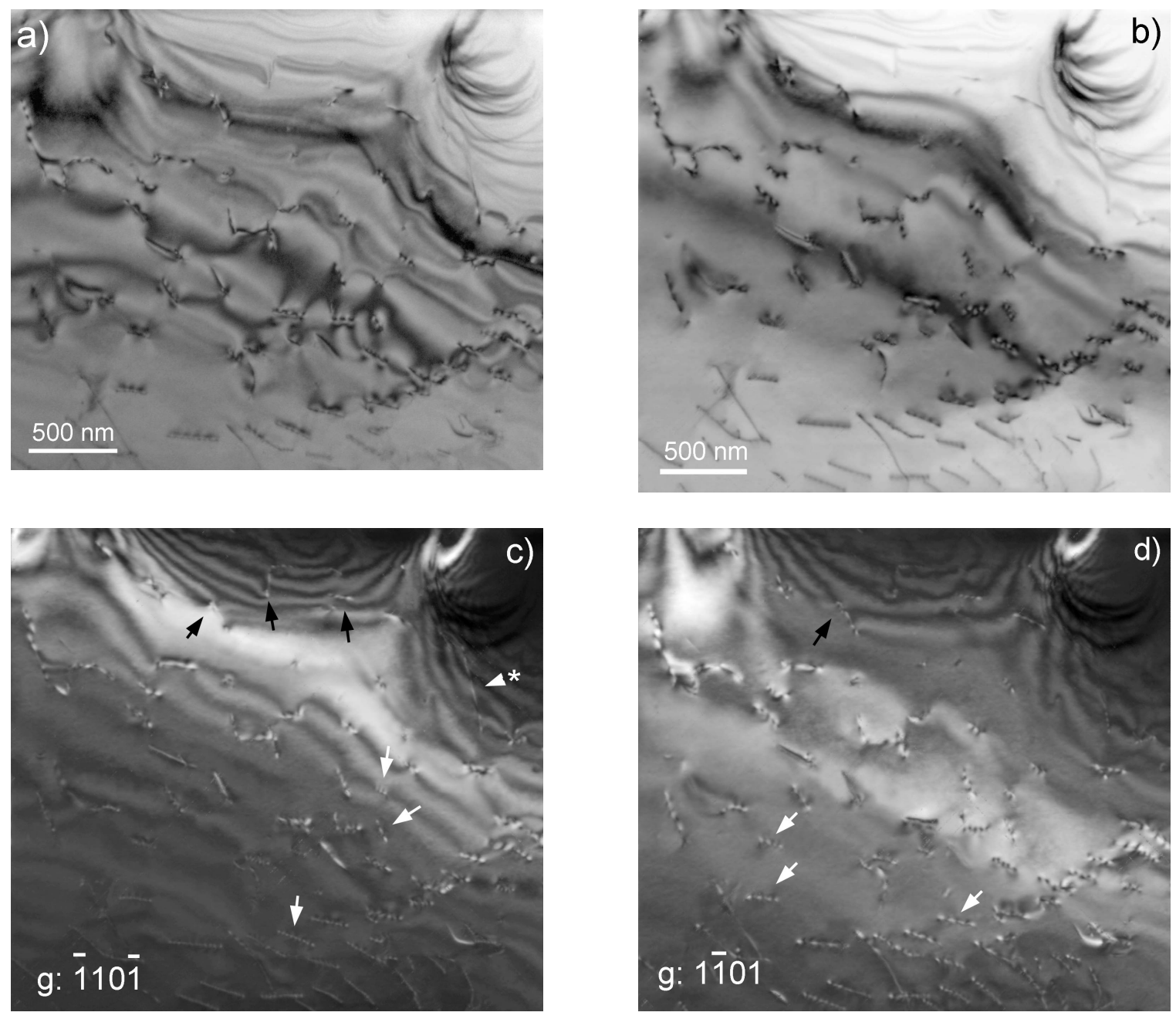

Figure 4: Quartz - Acquisition parameters: spot size $1 \mathrm{~nm}$; step size $2 \mathrm{~nm}$; acquisition time $30 \mathrm{~ms} ; 1500 \times 1500$ points. The sample is oriented close to the [1̄120] zone axis.

a) Conventional TEM bright-field image.

b) Virtual bright-field

c-d) Virtual dark-fields with $\{1 \overline{1} 01\}$

(For explanation of arrowed features, see text) 
Fig. 4 shows a VBF and two VDF images reconstructed with two $\{1 \overline{1} 01\}$ reflexions present in a [11 $\overline{2} 0]$ zone axis. These images are compared with a conventional bright-field image of the same area which has been recorded after the SED acquisition. It is seen that the 30 ms acquisition time (electron dose 10500 electrons.nm $\left.{ }^{2}\right)$ per pixel does not introduce any significant damage. One can see in Fig. 4 that all characteristic diffraction contrasts of conventional TEM are well reproduced. This shows that despite the diffraction patterns are recorded with a rather low resolution $(144 \times 144$ pixels $)$ to optimize the acquisition, their quality is sufficient to reconstruct images that carry the most important features. In particular, one can clearly see the thickness contours (upper part of Fig. 4c-d) and how they interact and terminate on dislocations (black arrows) as described by Ishida et al. (1980). This contrast helps in recognizing that the dislocation arrowed with a star is out of contrast: thickness contour fringes do not interact with it and cross its line. Its Burgers vector is thus $\pm_{\overline{3}} \frac{1}{1}[\overline{1} 20]$. The oscillatory contrast of dislocation lines inclined with respect to the foil mean plane is also clearly recognized (white arrows).

Fig. 5 shows the results from another acquisition in the same thin foil, again with an orientation close to the

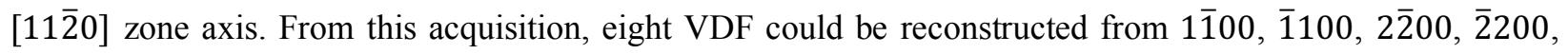
$1 \overline{1} 01, \overline{1} 10 \overline{1}, 3 \overline{3} 01$ and $\overline{3} 30 \overline{1}$ diffraction spots. This area displays numerous perfect dislocations which exhibit the same diffraction contrasts as discussed above. One also notices the presence of tiny dislocation loops (as commonly observed in wet quartz, either synthetic or natural, see Cordier et al., 1988). When the diameter of a dislocation loop is much larger than the extinction distance $\xi_{g}$, the loop appears as a closed dislocation line (Hirsch et al., 1960). Otherwise, it usually appears as a strain contrast characterized by a pair of black and white lobes (see examples arrowed on Fig. 5c and f; enlargements of loop $\alpha$ are displayed Fig. 6a and b). In case of a symmetrical dilation centre (coherent precipitate for instance), the line of no contrast which separates the black and white lobes is always perpendicular to the diffraction vector (Rühle et al., 1965). In case of tiny dislocation loops, the strain field is not symmetrical and the orientation of the line of no contrast is determined by the one of the Burgers vector (Hirsch et al., 1960; Rühle et al., 1965). This situation is illustrated by the defect labelled $\alpha$ (labelled in Figs $5 \mathrm{c}$ and $\mathrm{f}$ and for which enlargements are presented Figs $6 \mathrm{a}$ and $\mathrm{b}$ ): its contrast is reversed between Figs $6 a$ and $b$, but the line of no contrast keeps the same orientation although different diffraction vectors are involved. Further evidence is provided by Fig. 5i where the defect (black-and-white contrast) previously arrowed at the centre of Fig. $5 \mathrm{f}$ (labelled $\beta$ ) now clearly appears as a dislocation loop with a diameter of $20 \mathrm{~nm}$ (see enlargements in Figs 6c and d). Quite high resolutions are obtained, since on Fig. 5e, the dislocation loop arrowed exhibits a diameter of $16 \mathrm{~nm}$. It is to be remembered that this is the size of the image and not of the loop itself since the contrast of a dislocation line does not coincide with its actual position. This is illustrated when $+\boldsymbol{g}$ is reversed to $-\boldsymbol{g}$ as in Figs $5 \mathrm{~g}$ and $\mathrm{h}$ (enlargements in Figs 6e and f) where the loop arrowed $(\gamma)$ exhibits an apparent diameter of 30 and $18 \mathrm{~nm}$ respectively. All these observations reproduce very well the contrasts expected in conventional TEM. 

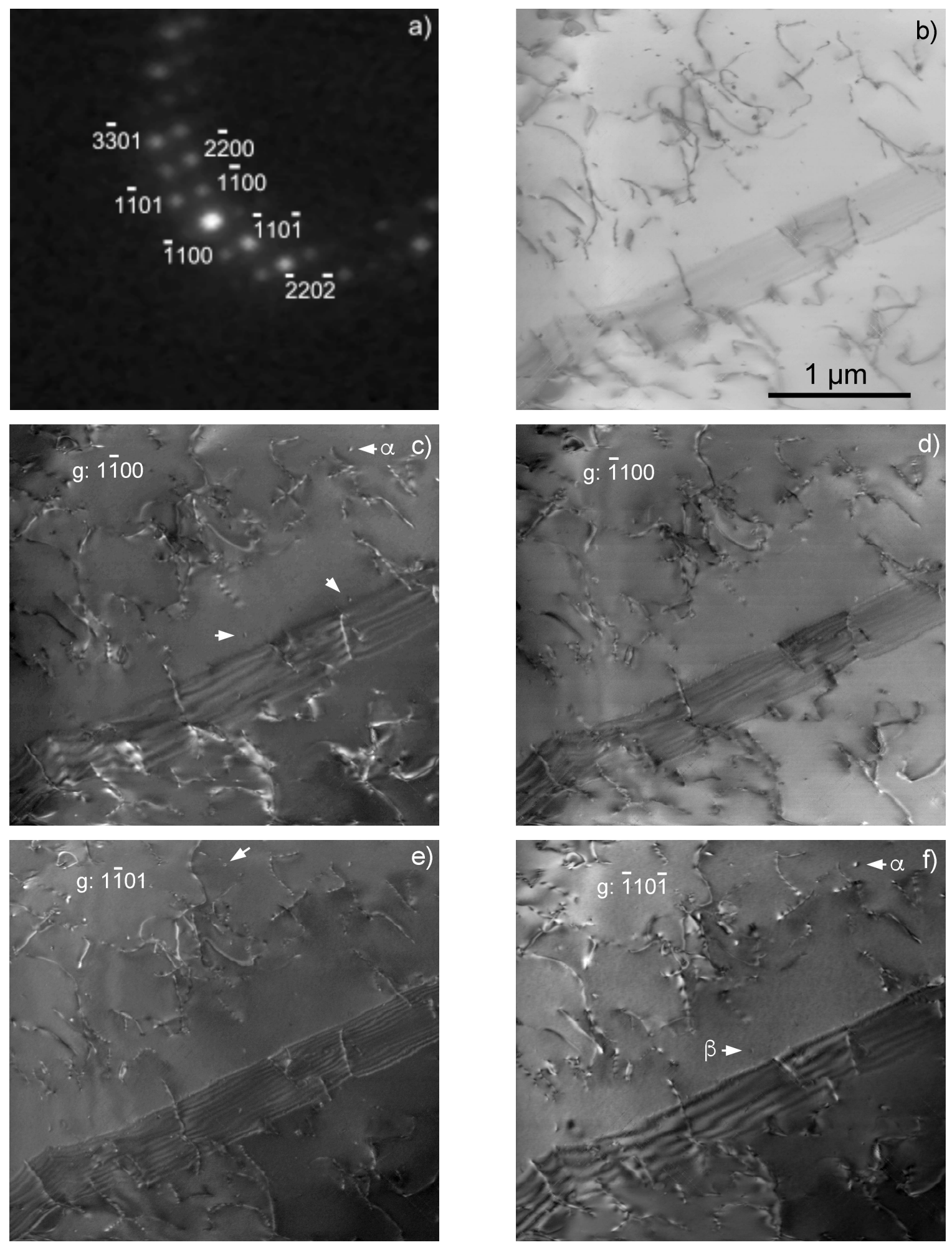

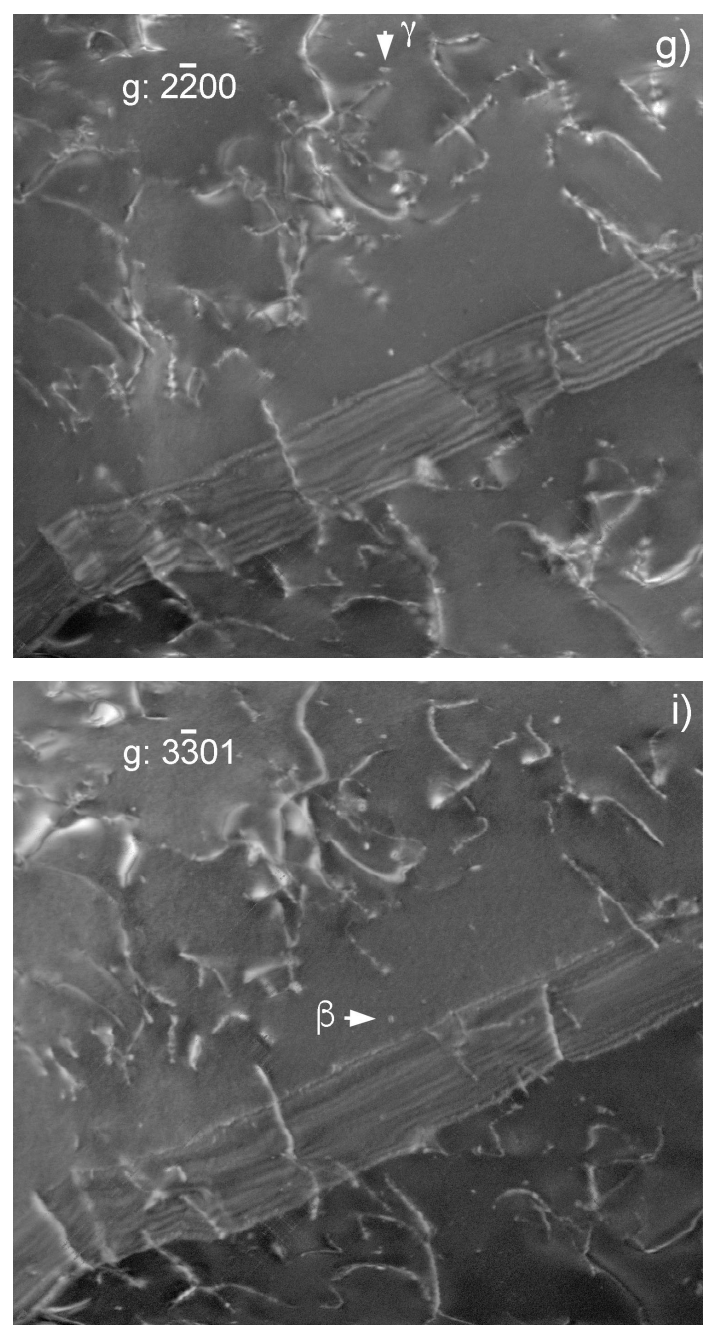
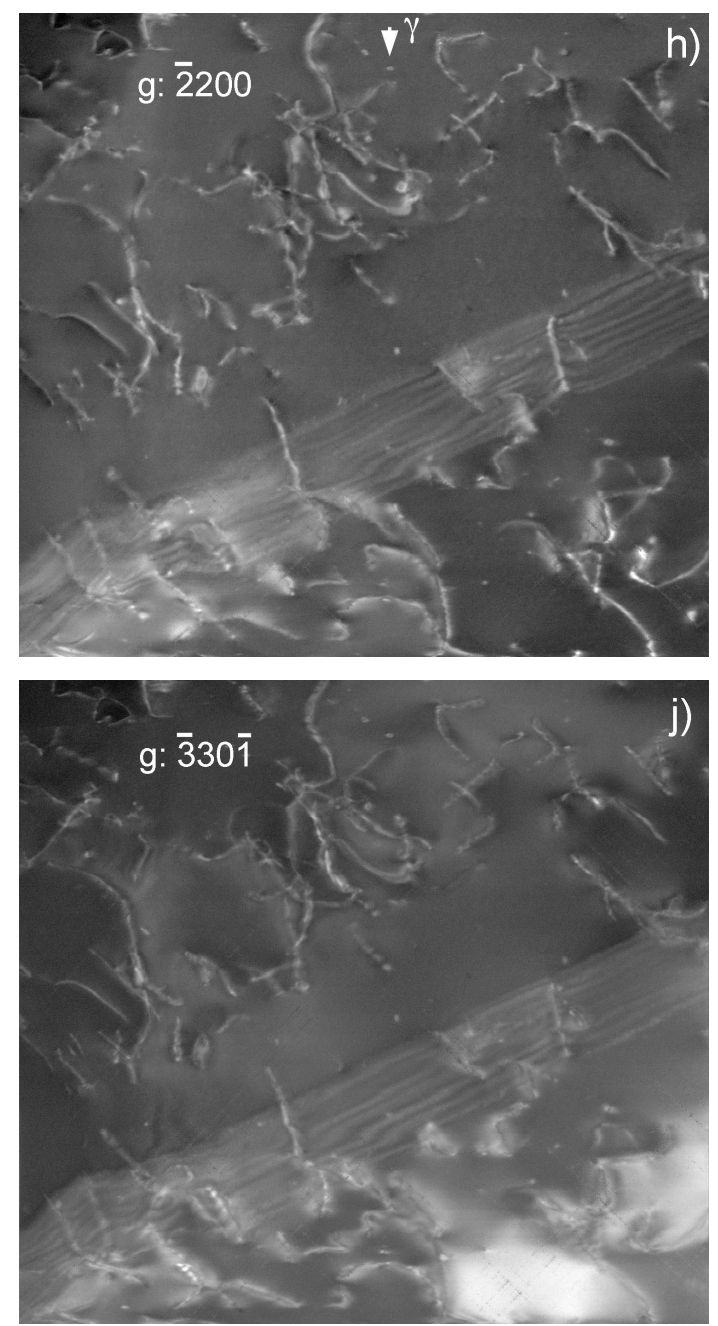

Figure 5: Quartz - Acquisition parameters: spot size $1 \mathrm{~nm}$; step size $2 \mathrm{~nm}$; acquisition time $30 \mathrm{~ms} ; 1500 \times 1500$ points.

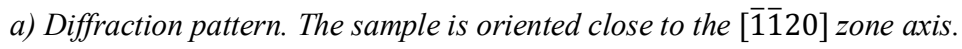

b) Virtual bright-field

c-j) Virtual dark-fields: the diffraction vector is indicated on the pictures

Fig. 5 also shows some distinctive fringe contrasts which are commonly observed in quartz in relation with plastic deformation and microtwinning. The two most common types of microtwins observed in quartz are related to the Dauphiné and Brazil twin laws. Dauphiné twin corresponds to a rotation of $180^{\circ}$ about the $c$-axis whereas Brazil twins are linked to the enantiomorphism of quartz which can belong to the $P 3_{1} 21$ (where $\mathrm{SiO}_{4}$ tetrahedra on the three-fold axes form a left-handed-helix) or to the $P 3_{2} 21$ (right-handed helix) space groups. Brazil twins correspond to alternating left-handed and right-handed domains. Twin boundaries are usually made visible through fringes which arise because a phase shift $\alpha$ occurs across the boundary. The kinematic theory describing this contrast has been originally developed by Hirsch et al. (1960). It has been further extended to account for dynamical effects (van Landuyt et al., 1964, 1965) and applied specifically to microtwins in quartz 
by McLaren and Phakey $(1966,1969)$. In case of Dauphiné twinning, the phase shift is null across the boundary for $g:\{1 \overline{1} 00\}$ and $g:\{1 \overline{1} 01\}$ and no diffraction contrast fringes are expected at the boundary (McLaren et al., 1969). Hence, Fig. 5 rather suggests boundaries corresponding to Brazil microtwins. Indeed, the displacement vector across a Brazil microtwin is of the $\frac{1}{6}\langle 11 \overline{2} 0\rangle$ type also involved in the dissociation of $\frac{1}{3}\langle 11 \overline{2} 0\rangle$ dislocations as discussed by Trépied and Doukhan (1978) and Doukhan (1995). The microstructure shown in Fig. 5 with multiple overlapping stacking faults and the presence of dislocations is consistent with this interpretation.
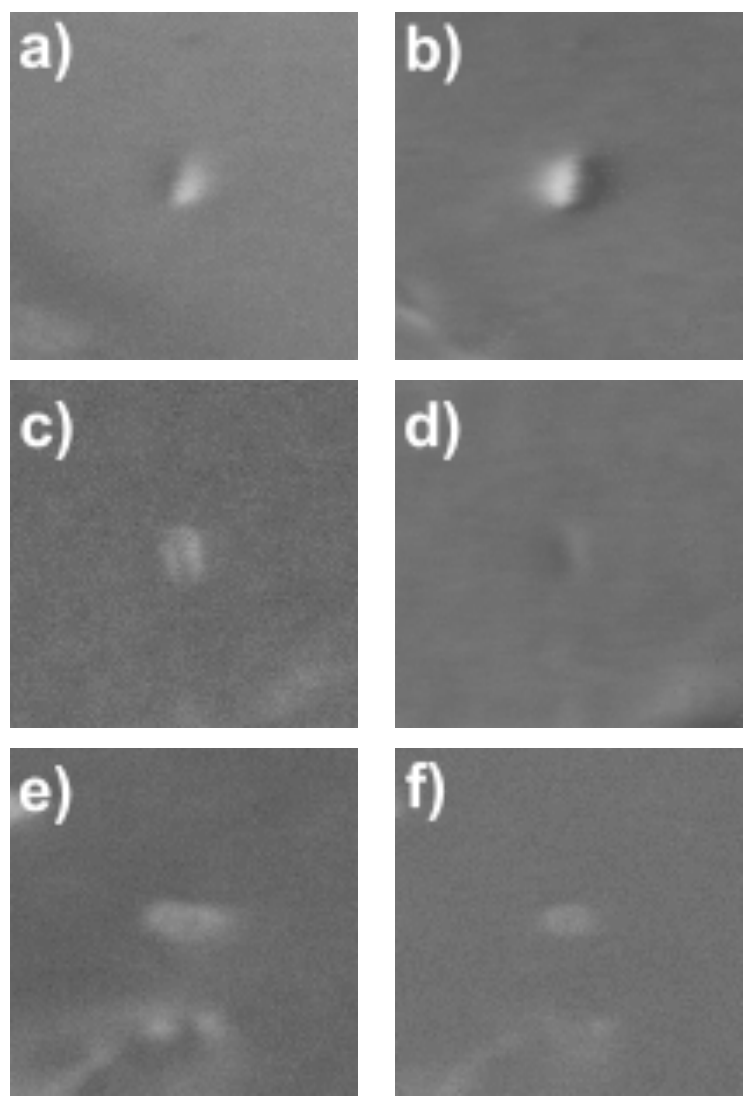

Figure 6: Quartz - Enlargements of Fig.5 showing some dislocation loops. All pictures are $100 \times 100$ pixels, i.e. $200 \mathrm{~nm}$ wide.

a) Loop a from Fig. $5 c-g: 1 \overline{1} 00$

b) Same loop as a), i.e. loop $\alpha$ from Fig. $5 f-g: \overline{1} 10 \overline{1}$

c) Loop $\beta$ from Fig. $5 i-g: 3 \overline{3} 01$

d) Same loop as c), i.e. loop $\beta$ from Fig. $5 f-g: \overline{1} 10 \overline{1}$

e) Loop $\gamma$ from Fig. $5 g-g: 2 \overline{2} 00$

f) Same loop as e), i.e. loop $\gamma$ from Fig. $5 h-g: \overline{2} 200$

The interpretation of diffraction contrast fringes is more straightforward at exact Bragg conditions $s=0$ (van Landuyt et al., 1964). In that case, for fringes corresponding to $\alpha=\pi$ (which is the case for Brazil microtwins imaged with $g:\{1 \overline{1} 00\}$ see McLaren et al. 1966), the fringe profiles are expected to be symmetrical with respect to the centre of the foil, and the bright- and dark-field images are complementary. This analysis is not very 
conclusive here. The (virtual) bright-field image does not correspond to two-beam conditions and it is difficult to infer which reflexion influences the contrast more. Concerning the dark-field images, as already underlined above, the fact that data acquisitions are performed at a constant tilt excludes a fine control of the Bragg deviation vectors. Given the local bending of the foils, it varies from place to place and from a virtual dark-field to another. Indeed, van Landuyt et al. (1964) have shown that deviation from the exact Bragg orientation can destroy the symmetry of the dark-field image. This may be the reason of the asymmetry of the fringes observed in Fig. $5 \mathrm{~d}$.

\section{Phase A}

Phase $\mathrm{A}\left(\mathrm{Mg}_{7} \mathrm{Si}_{2} \mathrm{O}_{8}(\mathrm{OH})_{6}\right)$ is a dense hydrous magnesium silicate (DHMS) phase which is supposed to form in cold hydrous parts of the subducting slabs, between 200 and $350 \mathrm{~km}$ depths, by destabilization of $\mathrm{Mg}$ phyllosilicates. Because of its chemical composition, phase A is likely to be the main water carrier at these depths and is viewed as a key phase for water transfer down to the transition zone in cold environments (e.g. Poli and Schmidt, 1997).
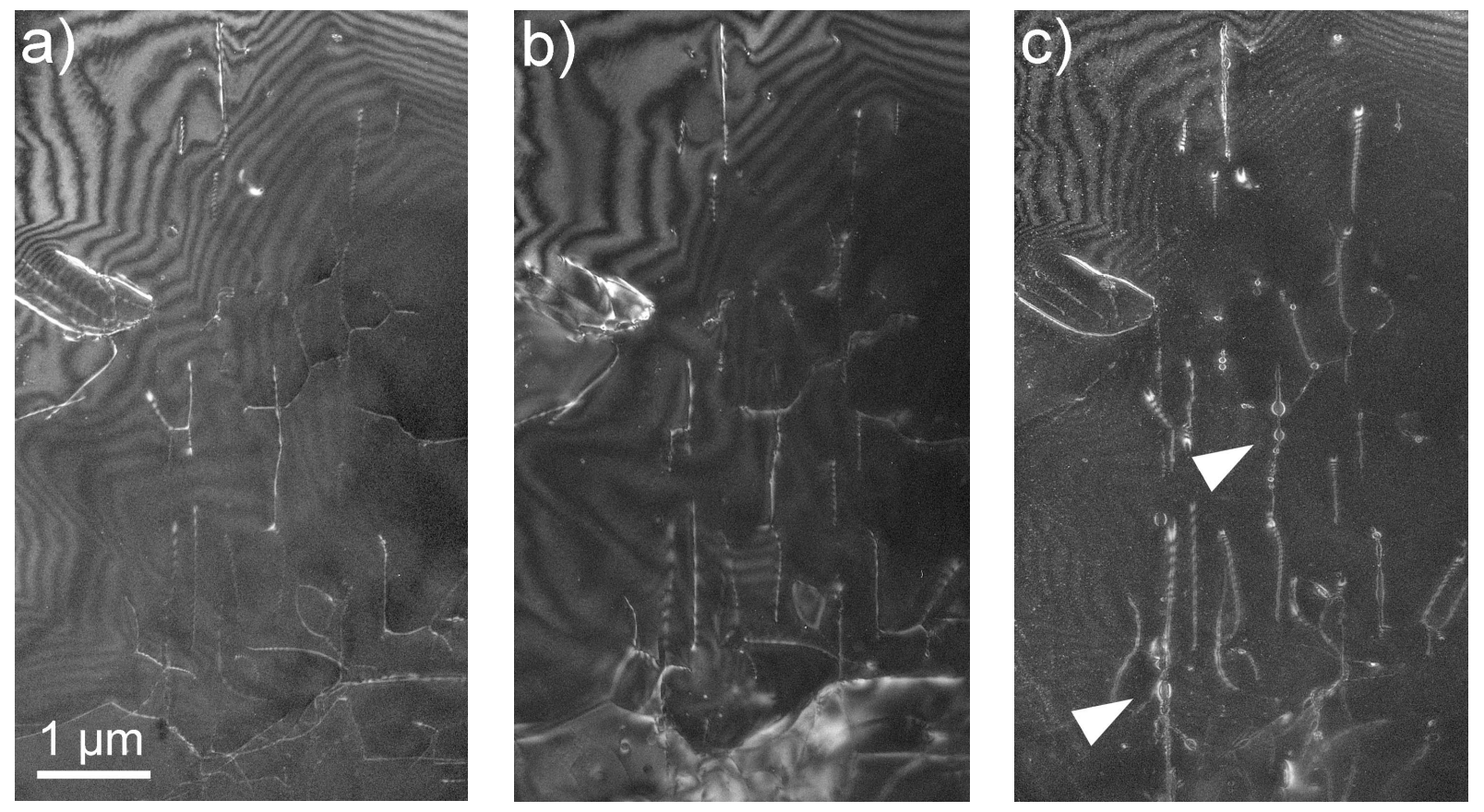

Figure 7: Phase A-Conventional TEM weak-beam dark-field images. The sample is oriented close to the [112̄0] zone axis. Observation conditions were performed to minimize beam damage, i.e. by using a small condenser aperture (100 $\mu \mathrm{m})$, a small spot size $(300 \mathrm{~nm})$, a low magnification $(M=3900 \times$ using photographic plates for further enlargement) and $a$ Gatan $^{\mathbb{B}}$ cooling TEM holder operated with liquid nitrogen $\left(T \approx-160^{\circ} \mathrm{C}\right.$ near the thin foil). Acquisition time is approximately $10 \mathrm{~s}$ for each micrograph.

a) $g: \overline{2} 20 \overline{2}$

b) $g: 2 \overline{2} 0 \overline{2}$

c) g: $000 \overline{8}$. Note the spotty background and the formation of tiny loops on the dislocation lines (arrowed) due to beam damage after c.a. 3 min of observation on this area. 
The sample studied here has been synthesized and deformed in the multianvil apparatus at the Bayerisches Geoinstitut, Bayreuth, Germany (Mussi et al., 2012). Phase A was synthesized from high purity oxides at 11 $\mathrm{GPa}$ (nominal pressure) and $900^{\circ} \mathrm{C}$. The recovered samples were further deformed at $11 \mathrm{GPa}$ and $700^{\circ} \mathrm{C}$ (run H3008). The first characterizations of crystal defects in phase A, performed by Mussi et al. (2012) has shown that phase A was very sensitive to electron beam damage and TEM characterization could only be performed under conditions adjusted to slow down beam damage, in particular using a liquid nitrogen cooling TEM holder. The structure of phase A is hexagonal, space group $P 6_{3}$, with $a=7.8603 \AA$ and $c=9.5730 \AA$ (Horiuchi et al., 1979). These larger large unit cell parameters put strong constraints on the dislocation Burgers vectors (see a computational study in Gouriet et al., 2015). So far, perfect dislocations observed exhibit Burgers vectors of the $\frac{1}{3}\langle 11 \overline{2} 0\rangle$ (so called $<\mathrm{a}>$ ) and $\frac{1}{3}\langle 11 \overline{2} 3\rangle$ (so called $<\mathrm{c}+\mathrm{a}>$ ) types (Mussi et al., 2012). To perform these characterizations, the dislocations must be imaged with different diffraction vectors. This requires spending some time on the same area to adjust several diffracting conditions. Figure 7 demonstrates that even when maximum care is taken to minimize beam damage like using a cold stage and low illumination (experimental conditions are detailed in the figure caption), beam damage occurs very rapidly with this phase. This is shown by the spotty aspect of the background (well visible on the thickness fringes at the top left of Fig. 7c) or more severely by the many dislocation loops which grow on the dislocations (white arrows on Fig. 7c), which form probably by condensation of point defects resulting from electron irradiation. Figure 8, obtained on the same foil, shows that SED represents an attractive alternative for those phases. Here a standard sample holder is used (not cooled). The foil was simply oriented for the grain under study to be oriented close to a convenient zone axis. Here [ $5 \overline{4} \overline{1} 0]$ is chosen which contains reflections with high structure factors (1395 for 0008; 1057 for $12 \overline{3} 5 ; 12 \overline{3} 2$ and $12 \overline{3} 3$ exhibit values of 350 and 163 respectively; the lowest structure factor among those presented is 49 for $24 \overline{6} 0$ ) which are adapted for Burgers vectors characterization. With the sole acquisition leading to the five VDF presented in Fig. 8, it is possible from the intensity profiles to identify (see supplementary materials) a $\frac{1}{3}[1 \overline{2} 10]$ dislocation (labelled A on Fig. 8) and a $\frac{1}{3}[11 \overline{2} 3]$ dislocation (labelled B on Fig. 8). No evidence for beam damage was observed on the mapped area after acquisition. 
a)
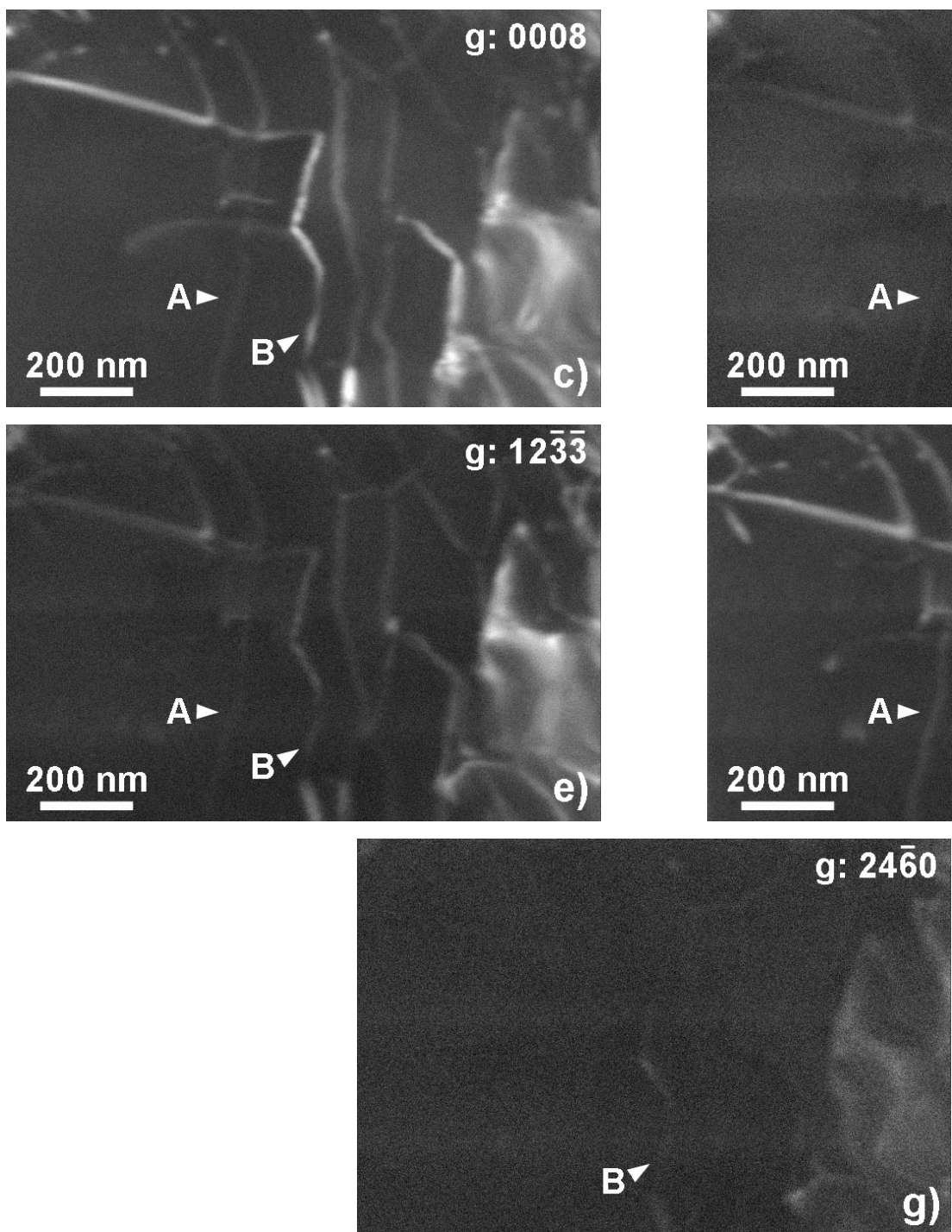

Figure 8: Phase A-Acquisition parameters: spot size $1 \mathrm{~nm}$; fluence rate $3.5 \times 10^{5}$ electron. $\mathrm{nm}^{-2} \mathrm{~s}^{-1}$; step size $2 \mathrm{~nm}$; acquisition time $60 \mathrm{~ms}$. The images presented are cropped from a map recorded with $700 \times 700$ points.

a) Diffraction pattern. The sample is oriented close to the [5 $\overline{4} \overline{1} 0]$ zone axis.

b) Indexation of the diffraction pattern highlighting the diffraction vectors utilized.

c-g) Virtual dark-fields: the diffraction vector is indicated on the pictures. 


\section{Conclusions}

Images presenting all characteristics of bright- and dark field images from conventional TEM can be reconstructed from the data cube of diffraction patterns obtained from SED. The image quality and resolution of dislocations (diffraction contrast of strain fields) are comparable with those obtained from conventional TEM. The main advantage is the possibility of extracting a large number of images from different diffraction vectors based on a single acquisition with a very short exposure time per pixel. This possibility is very attractive for investigating beam sensitive materials as illustrated here with quartz and phase A.

The technique has however some drawbacks. The acquisition of images with a high spatial resolution (here 2 $\mathrm{nm}$ ) is long (for quartz, $1500 \times 1500$ images with an acquisition time of $30 \mathrm{~ms}$ correspond to more than eighteen hours and for phase A, 700×700 images with an acquisition time of $60 \mathrm{~ms}$ correspond to more than eight hours). However, a major limitation is related to the fact that all VDF correspond to a single orientation. In diffraction contrast, very small tilt adjustments are always necessary to precisely adjust the Bragg deviations in order to optimize the contrast. This is not possible here and our examples show that, at a given location, the diffraction conditions are not precisely comparable from one image to another. This might be a limitation for some precise contrast interpretation (as shown with dislocations and fringe contrasts related to interfaces).

Despite these limitations, we consider that SED represents a very powerful technique which complements our possibilities of characterizing defects in beam-sensitive materials.

\section{Acknowledgements}

This work was supported by funding from the European Research Council under the Seventh Framework Programme (FP7), ERC grant N²90424 - RheoMan to P. Cordier. The TEM facility in Lille (France) is supported by the Conseil Regional du Nord-Pas de Calais, and the European Regional Development Fund (ERDF). We thank the reviewers and the associate editor for their useful comments on the manuscript.

\section{References}

Barnard JS, Sharp J, Tong JR and Midgley PA (2006a) High-resolution three-dimensional imaging of dislocations. Science, 313, 319.

Barnard JS, Sharp J, Tong JR and Midgley PA (2006b) Three dimensional analysis of dislocation networks in GaN using weak-beam dark-field electron tomography. Philosophical Magazine, 86, 4901-4922.

Barnard JS, Eggeman AS, Sharp J, White TA and Midgley PA (2010) Dislocation electron tomography and precession electron diffraction - minimising the effects of dynamical interactions in real and reciprocal space. Philosophical Magazine, 90, 4711-4730 
Cockayne DJH, Ray ILF and Whelan MJ (1969) Investigation of dislocation strain fields using weak beams Philosophical Magazine 20, 1265-1270.

Cordier P, Boulogne B and Doukhan JC (1988) Water precipitation and diffusion in wet quartz and wet berlinite $\mathrm{AlPO}_{4}$. Bulletin de Minéralogie, 111, 113-137

Cordier P, Morniroli JP and Cherns D (1995) Characterization of crystal defects in quartz by large-angle convergentbeam electron diffraction. Philosophical Magazine 72, 1421-1430.

Doukhan JC (1995) Lattice Defects and Mechanical Behaviour of Quartz $\mathrm{SiO}_{2}$. Journal de Physique III, 5, 1809-1832.

Gouriet, K., Hilairet, N., Amiguet, E., Bolfan-Casanova, N., Wang, J., Reynard, B. and Cordier, P. (2015) Plasticity of the dense hydrous magnesium silicate Phase A at subduction zones conditions. Physics of the Earth and Planetary Interiors, 248, 1-11.

Hirsch PB, Horne RW and Whelan MJ (1956) Direct observation of the arrangement and motion of dislocations in aluminium. Philosophical Magazine 1, 677-684.

Hirsch PB, Howie A and Whelan MJ (1960) A kinematical theory of diffraction contrast of electron transmission microscope images of dislocations and other defects. Philosophical Transactions of the Royal Society A252, 499529.

Horiuchi, H., Morimoto, N., Yamamoto, K., Akimoto, S.I. (1979) Crystal-structure of $2 \mathrm{Mg}_{2} \mathrm{SiO}_{4} .3 \mathrm{Mg}(\mathrm{OH})_{2}$, a new high-pressure structure type. American Minereralogist, 64, 593-598.

Ishida Y, Ishida H, Kohra K and Ichinose H (1980) Determination of the Burgers vector of a dislocation by weakbeam imaging in a HVEM. Philosophical Magazine 42, 453-462.

Kiss, A.K., Rauch, E.F. and Lábár, J.L. (2016) Highlighting material structure with transmission electron diffraction correlation coefficient maps. Ultramicroscopy, 163, 31-37.

Lieven L, Prewitt CT and Weidner DJ (1980) Structure and elastic properties of quartz at pressure. American Mineralogist 65, 920-930.

McLaren AC and Phakey PP (1965a) Dislocations in Quartz observed by Transmission Electron Microscopy. Journal of Applied Physics 36, 3244-3246.

McLaren AC and Phakey PP (1965b) A transmission electron microscope study of amethyst and citrine. Australian Journal of Physics 18, 135-141.

McLaren AC and Phakey PP (1966) Electron microscope study of Brazil twin boundaries in amethyst quartz. Physica Status Solidi 13, 413-422.

McLaren AC and Phakey PP (1969) Diffraction contrast from Dauphiné twin boundaries in quartz. Physica Status Solidi 31, 723-737.

Mussi A, Cordier P and Frost, D.J. (2012) Crystal defects in dense hydrous magnesium silicate phase A deformed at high pressure: characterization by transmission electron microscopy. European Journal of Mineralogy, 24, 429438.

Mussi A, Cordier P, Demouchy S and Vanmansart C (2014) Characterization of the glide planes of the [001] screw dislocations in olivine using electron tomography. Physics and Chemistry of Minerals, 41, 537-545.

Poli S and Schmidt M. (1997) The high-pressure stability of hydrous phases in orogenic belts: an experimental approach on eclogite-forming processes. Tectonophysics, 273, 169-184

Rauch EF and Dupuy L (2005) Rapid spot diffraction patterns identification through template matching, Archives of Metallurgy and Materials, 50, 87-99.

Rauch EF and Véron M (2014a) Analyzing dislocations with virtual dark field images reconstructed from electron diffraction patterns. Microscopy and Microanalysis 20(Suppl 3), 1456-1457.

Rauch EF and Véron M (2014b) Automated crystal orientation and phase mapping in TEM. Materials Characterization, 98, 1-9. 
Rühle M, Wilkens M and Essmann U (1965) Zur deutung des elecktronenmikroskopischen kontrasterscheinungen an fehlstellenagglomeraten in neutronenbestrahltem kupfer. Physica Status Solidi, 11, 819-829.

Thieme M, Demouchy S, Mainprice D, Barou F, and Cordier P (2018) Stress evolution and associated microstructure during transient creep of olivine at $1000-1200^{\circ} \mathrm{C}$. Physics of the Earth and Planetary Interiors, 278, 34-46.

Trépied L. and Doukhan JC (1978) Dissociated 'a' dislocations in quartz. Journal of Materials Science, 13, 492-498.

Van Landuyt J, Gevers R and Amelinckx S (1964) Fringe patterns at anti-phase boundaries with $\alpha=\pi$ observed in the electron microscope. Physica Status Solidi, 7, 519-546.

Van Landuyt J, Gevers R and Amelinckx S (1965) Dynamical theory of the images of microtwins as observed in the electron microscope I. Overlapping twins. Physica Status Solidi, 9, 135-165. 